\title{
Interchange instability of a curved current layer convecting in the magnetosheath from the bow shock towards the magnetopause
}

\author{
I. L. Arshukova ${ }^{1}$, N. V. Erkaev $^{1}$, and H. K. Biernat ${ }^{2,3}$ \\ ${ }^{1}$ Institute of Computational Modelling, Russian Academy of Sciences, Krasnoyarsk-36, 660036, Russia \\ ${ }^{2}$ Space Research Institute, Austrian Academy of Sciences, Schmiedlstrasse 6, 8042 Graz, Austria \\ ${ }^{3}$ also at: Institute of Geophysics, Astrophysics, and Meteorology, University of Graz, 8010 Graz, Austria
}

Received: 14 February 2003 - Revised: 05 August 2003 - Accepted: 22 August 2003 - Published: 19 March 2004

\begin{abstract}
This paper deals with nonsteady perturbations of the magnetosheath parameters which are related to variations of the interplanetary magnetic field from north to south under a constant solar wind dynamic pressure. The magnetic field changes its direction within a thin layer which is convected with the plasma from the bow shock to the ionopause. In the course of time, this current layer is amplified during its motion towards the magnetopause. The intensity of the current is increasing, the layer thickness is decreasing, and the gradients of parameters are becoming much sharper while the layer is approaching the magnetopause. The curvature radius of this layer is decreasing while it is draping around the magnetopause. This curved layer structure with reversed magnetic field in the magnetosheath is found to be unstable with respect to the interchange instability. The growth rate of the instability is obtained for different positions of the layer.
\end{abstract}

Key words. Magnetospheric physics (magnetosheath)

\section{Introduction}

Solar wind conditions are very changeable, and in particular, the interplanetary magnetic field can reverse its direction. Tangential discontinuities carried by the solar wind are typical structures with such interplanetary magnetic field (IMF) variations. Horbury et al. (2001) analyzed solar wind discontinuities using three spacecraft observations, and they identified about $14 \%$ of the observations as tangential discontinuities which are characterized by large variations of the total magnetic field with respect to its maximum value. The time scale of a tangential discontinuity estimated from the example of Horbury et al. (2001) is less than $1 \mathrm{~min}$. It is rather difficult to determine the discontinuity scale precisely because of fluctuations of the magnetic field and plasma parameters on either side of a discontinuity.

Correspondence to: $\mathrm{H}$. K. Biernat

(helfried.biernat@kfunigraz.ac.at)
Thin current layers related to the tangential discontinuities are coming to the magnetosheath. They are moving towards the magnetopause, together with the ambient plasma. Approaching towards the magnetopause, these layers have a curvature radius being approximately equal to that of the magnetopause.

As shown by Arshukova and Erkaev (2000), a curved layer with reversal magnetic fields can be unstable with respect to the interchange instability if the plasma pressure has a local maximum which coincides with the magnetic pressure minimum within the layer. The interchange instability is similar in nature to the Rayleigh-Taylor instability in classical hydrodynamics, where the magnetic stress plays the role of an effective gravitational force (Chandrasekhar, 1968; Alexandrov et al., 1984; Freidberg, 1987). In the context of the Earth's magnetospheric boundary (magnetopause), the interchange instability was analyzed for a tangential discontinuity by Alexeev and Maltsev (1990), and also by Rezenov and Maltsev (1994). The MHD instabilities of structured plasma layers with piecewise constant parameters were studied by Arshukova et al. (2002).

The aim of our present paper is to study the interchange instability of a thin current layer with reversal magnetic fields which is comoving with plasma in the magnetosheath from the bow shock to the magnetopause. The paper is organized as follows: In the second section, a numerical solution is discussed with regard to a current layer moving from the bow shock to the magnetopause. In the third section, the statement of the instability problem is described, and a boundary value problem is formulated. In the fourth section, numerical results are described for the instability growth rate and calculated for two cases: 1) Instability of the layer with an analytical model profile of the magnetic field; 2) Instability of the layer in the magnetosheath with the magnetic field profiles obtained from the numerical solution of the solar wind flow problem in the case of a nonsteady IMF variation from north to south. 


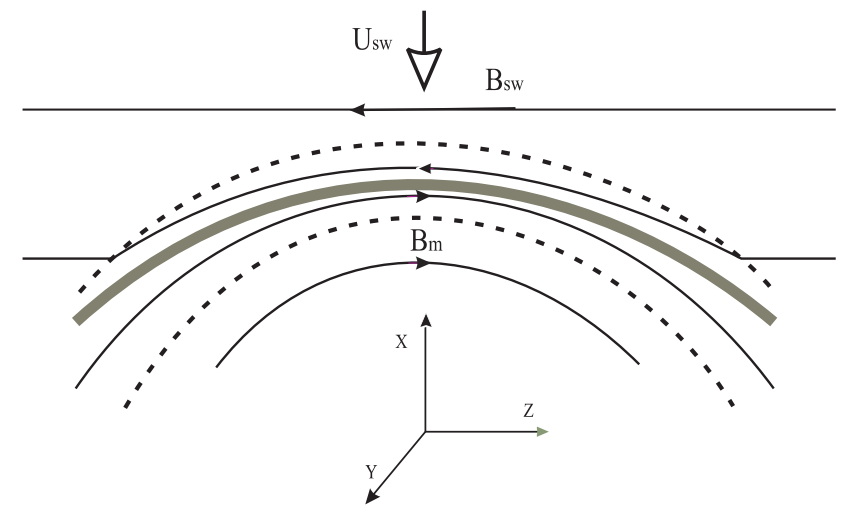

Fig. 1. General view of a curved current layer separating antiparallel magnetic fields.

\section{A current layer moving from the bow shock towards the magnetopause}

We assume that the IMF changes its direction from north to south in the solar wind upstream of the shock under a constant solar wind dynamic pressure. For this magnetic field variation, there exists a current layer which is convected from the bow shock towards the magnetopause (see Fig. 1). In this layer, the magnetic field is depressed and thus the density and plasma pressure are enhanced.

To describe time dependent magnetic field and plasma parameters in the magnetosheath, we apply the nonstationary ideal MHD equations which can be written as follows (Landau and Lifshitz, 1960),

$$
\begin{aligned}
& \rho \frac{\partial \mathbf{U}}{\partial t}+\rho(\mathbf{U} \cdot \nabla) \mathbf{U}+\nabla \Pi-\frac{1}{4 \pi}(\mathbf{B} \cdot \nabla) \mathbf{B}=0, \\
& \frac{\partial \rho}{\partial t}+\nabla \cdot(\rho \mathbf{U})=0, \quad \frac{\partial}{\partial t}\left(\frac{P}{\rho^{\kappa}}\right)+\mathbf{U} \nabla\left(\frac{P}{\rho^{\kappa}}\right)=0, \\
& \frac{\partial \mathbf{B}}{\partial t}-\nabla \times(\mathbf{U} \times \mathbf{B})=0, \quad \nabla \cdot \mathbf{B}=0, \\
& \Pi=P+B^{2} /(8 \pi) .
\end{aligned}
$$

For our instability analysis, we use the profiles of the magnetic field and plasma parameters which are obtained from the MHD model of a nonsteady solar wind flow around the magnetosphere (Erkaev et al., 2002) modeled as a paraboloid of revolution. This model is similar to that applied previously to a steady solar wind flow around the Earth's magnetosphere (Farrugia et al., 1995), or the nonsteady flow around a magnetic cloud (Erkaev et al., 1995). In this model, the total pressure (4) is approximated by the Newtonian formula (Petrinec and Russell, 1987).

Figure 2 shows from top to bottom, the normalized profiles of plasma density, magnetic field, plasma pressure and magnetic pressure which correspond to different times (in units of $R / U_{s w}$ ). The normalization factors for the plasma density, magnetic field and pressures are $\rho_{s w}, \sqrt{4 \pi \rho_{s w}} U_{s w}$,
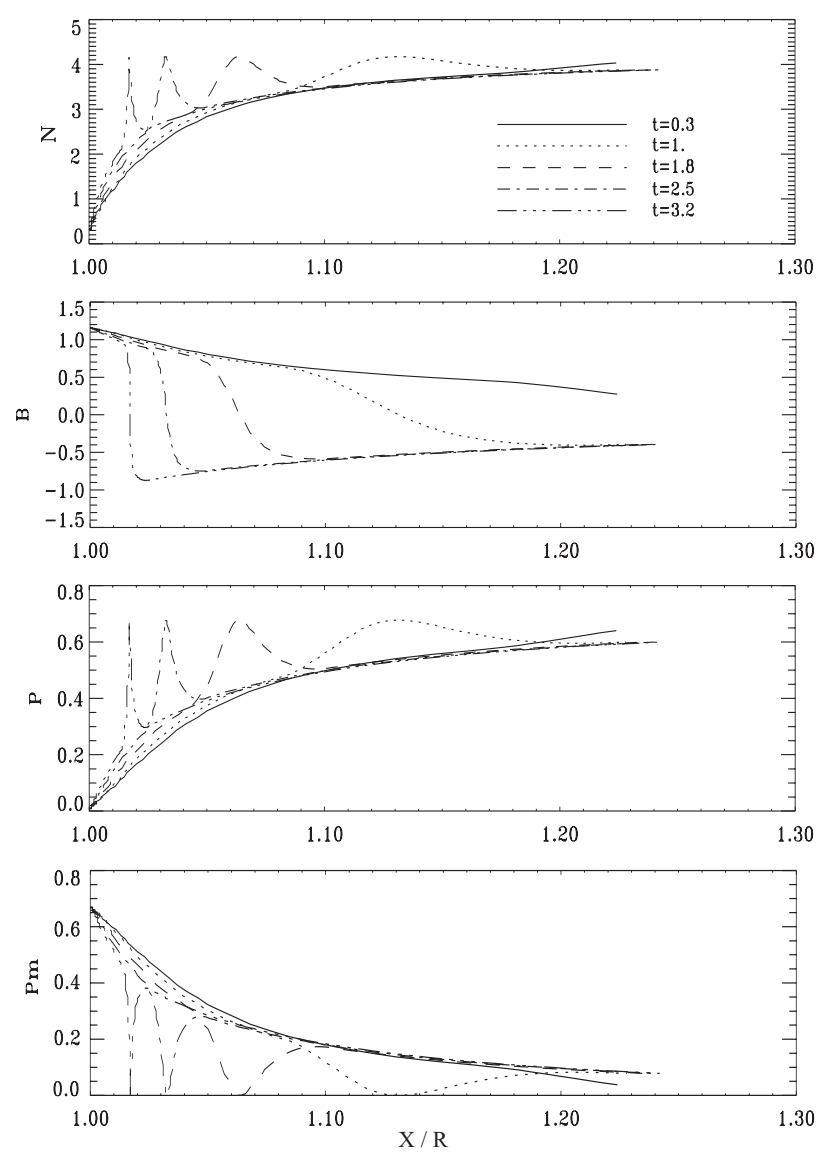

Fig. 2. Nonsteady profiles of plasma density, magnetic field strength, plasma pressure and magnetic pressure along the subsolar line.

and $\rho_{s w} U_{s w}^{2}$, respectively. The figure indicates a thin layer related to a nonsteady variation of IMF which is moving slowly towards the magnetopause. The layer is characterized by a local enhancement of the plasma pressure and density. While the layer is moving towards the magnetopause, the layer thickness is decreasing, the curvature radius is slightly decreasing, and the magnetic field strength is increasing with saturation.

It is evident that a steepening of the front of the magnetic field variation takes place, while it is approaching the magnetopause. This front of a large magnetic field gradient is characterized by enhanced electric current density, which, increases greatly while the front is moving towards the magnetopause.

The behavior of the layer, as shown in Fig. 2, indicates that magnetosheath flow structures provide nonlinear transformations and amplifications of smooth solar wind perturbations occurring under a constant solar wind dynamic pressure. Gradients of perturbations of the magnetic field, plasma pressure, and density are increasing very strongly while the perturbations are being convected towards the magnetopause. In particular, this can be the reason for slow mode perturbations observed in the magnetosheath (Song et al., 1992). The 
variation of the IMF from north to south brings about a transition current layer which is convecting from the bow shock towards the magnetopause. The thickness of this layer, as well as the amplitude of the current density, increase very strongly in the magnetic barrier.

\section{Statement of the instability problem}

Generally, a curved layer is characterized by two main local curvature radii, $R_{y}$ and $R_{z}$. We introduce a local coordinate system related to a surface within the layer. The two coordinates $y$ and $z$ are the distances along the geodetic lines on the surface with curvature radii $R_{y}$ and $R_{z}$, respectively. The third coordinate $x$ is the distance along the normal to the surface.

Let us consider small perturbations of the magnetic field and plasma parameters

$\mathbf{B}=\mathbf{B}^{*}+\mathbf{b}, \quad \mathbf{U}=\mathbf{U}^{*}+\mathbf{u}$,

$\Pi=P^{*}+p, \quad \rho=\rho^{*}+\rho^{\prime}$,

where $|\mathbf{b}| \ll|\mathbf{B}|,|\mathbf{u}| \ll|\mathbf{U}|, p \ll P$, and $\rho^{\prime} \ll \rho$. We study incompressible modes of perturbations and thus assume

$\nabla \cdot \mathbf{u}=0$.

The background plasma velocity $U^{*}$, as well as the normal component $B_{x}^{*}$ of the background magnetic field, are assumed to be equal to zero. Using these conditions, we obtain from (1) the following equations for small perturbations of the velocity components:

$\frac{\partial u_{x}}{\partial t}+\frac{1}{\rho} \frac{\partial p}{\partial x}=\frac{1}{4 \pi \rho}\left(\left(\mathbf{B}^{*} \cdot \nabla^{*}\right) b_{x}-2\left[\frac{B_{y}^{*} b_{y}}{q_{y} R_{y}}+\frac{B_{z}^{*} b_{z}}{q_{z} R_{z}}\right]\right)$,

$\frac{\partial u_{y}}{\partial t}+\frac{1}{q_{y} \rho} \frac{\partial p}{\partial y}=\frac{1}{4 \pi \rho}\left(\left(\mathbf{B}^{*} \cdot \nabla^{*}\right) b_{y}+\frac{B_{y}^{*} b_{x}}{q_{y} R_{y}}+b_{x} \frac{\partial B_{y}^{*}}{\partial x}\right)$,

$\frac{\partial u_{z}}{\partial t}+\frac{1}{q_{z} \rho} \frac{\partial p}{\partial z}=\frac{1}{4 \pi \rho}\left(\left(\mathbf{B}^{*} \cdot \nabla^{*}\right) b_{z}+\frac{B_{z}^{*} b_{x}}{q_{z} R_{z}}+b_{x} \frac{\partial B_{z}^{*}}{\partial x}\right)$,

$\frac{\partial P^{*}}{\partial x}=-\frac{1}{4 \pi}\left(\frac{B_{y}^{* 2}}{q_{y} R_{y}}+\frac{B_{z}^{* 2}}{q_{z} R_{z}}\right)$.

Here, $\nabla^{*}$ is a vector operator defined as

$$
\nabla^{*}=\left(\frac{\partial}{\partial x}, \frac{1}{q_{y}} \frac{\partial}{\partial y}, \frac{1}{q_{z}} \frac{\partial}{\partial z}\right)
$$

where $q_{y}$ and $q_{z}$ are the metric coefficients related to the curvature $q_{y}=1+x / R_{y}$ and $q_{z}=1+x / R_{z}$.

Initially, the plasma is assumed to satisfy quasi-steady conditions and thus, a gradient of the total pressure has to compensate the magnetic stress.

From (3) we obtain equations for small perturbations of the magnetic field components,

$$
\frac{\partial b_{x}}{\partial t}=\left(\mathbf{B}^{*} \cdot \nabla^{*}\right) u_{x},
$$

$$
\begin{aligned}
& \frac{\partial b_{y}}{\partial t}=\left(\mathbf{B}^{*} \cdot \nabla^{*}\right) u_{y}+u_{x} B_{y}^{*} \frac{1}{q_{y} R_{y}}-u_{x} \frac{\partial B_{y}^{*}}{\partial x}, \\
& \frac{\partial b_{z}}{\partial t}=\left(\mathbf{B}^{*} \cdot \nabla^{*}\right) u_{z}+u_{x} B_{z}^{*} \frac{1}{q_{z} R_{z}}-u_{x} \frac{\partial B_{z}^{*}}{\partial x}, \\
& \frac{\partial \rho^{\prime}}{\partial t}+u_{x} \frac{\partial \rho^{*}}{\partial x}=0 .
\end{aligned}
$$

For simplicity, we consider the two curvature radii to be equal to each other, $R_{y}=R_{z}=R$. For computational convenience, we introduce the dimensionless parameters

$\tilde{x}=x / R, \quad \tilde{\mathbf{k}}=\mathbf{k} R, \quad \tilde{\rho}=\rho / \rho_{s w}$,

$\tilde{\mathbf{B}}=\frac{\mathbf{B}}{\sqrt{4 \pi \rho_{s w} U_{s w}^{2}}}, \quad \tilde{\mathbf{U}}=\mathbf{U} / U_{s w}, \quad \tilde{p}=\frac{p}{\rho_{s w} U_{s w}^{2}}$,

$\tilde{\omega}=\omega R / U_{s w}, \quad \tilde{t}=t U_{s w} / R$.

Here, the subscript " $s w$ " denotes solar wind parameters.

Near the subsolar line, the coefficients of the equations for the perturbations are assumed to be functions of the normal distance $x$ only. Considering all perturbations to be proportional to the complex exponential function $\exp (i(\mathbf{k} \cdot \mathbf{s}-\tilde{\omega} t))$, where $\mathbf{s}$ is a two-dimensional vector in the plane $(y z)$, we obtain from Eqs. (7) and $\nabla \cdot \mathbf{u}=0$,

$$
\begin{aligned}
& -i q \tilde{\rho^{*}} \tilde{\omega} \tilde{u}_{x}+q \frac{\partial \tilde{p}}{\partial \tilde{x}}=i(\tilde{\mathbf{B}} \cdot \tilde{\mathbf{K}}) \tilde{b}_{x}-2(\tilde{\mathbf{B}} \cdot \tilde{\mathbf{b}}), \\
& -q \tilde{\rho}^{*} \tilde{\omega} \tilde{u}_{y}+\tilde{k}_{y} \tilde{p}=(\tilde{\mathbf{B}} \cdot \tilde{\mathbf{k}}) \tilde{b}_{y}-i \tilde{b}_{x}\left(\tilde{B}_{y}+q \frac{\partial \tilde{B}_{y}}{\partial \tilde{x}}\right), \\
& -q \tilde{\rho}^{*} \tilde{\omega} \tilde{u}_{z}+\tilde{k}_{z} \tilde{p}=(\tilde{\mathbf{B}} \cdot \tilde{\mathbf{k}}) \tilde{b}_{z}-i \tilde{b}_{x}\left(\tilde{B}_{z}+q \frac{\partial \tilde{B}_{z}}{\partial \tilde{x}}\right), \\
& \frac{\partial \tilde{u}_{x}}{\partial \tilde{x}}+\frac{1}{q} i(\tilde{\mathbf{u}} \cdot \tilde{\mathbf{k}})+\frac{2}{q} \tilde{u}_{x}=0,
\end{aligned}
$$

where $q=q_{y}=q_{z}=1+\tilde{x}$. After normalization, the system of the Eqs. (8-10) yields

$i q \tilde{\omega} \tilde{b}_{x}=-i(\tilde{\mathbf{B}} \cdot \tilde{\mathbf{k}}) \tilde{u}_{x}$,

$q \tilde{\omega} \tilde{b}_{y}=-(\tilde{\mathbf{B}} \cdot \tilde{\mathbf{k}}) \tilde{u}_{y}+i \tilde{u}_{x}\left(\tilde{B}_{y}-q \frac{\partial \tilde{B}_{y}}{\partial \tilde{x}}\right)$,

$q \tilde{\omega} \tilde{b}_{z}=-(\tilde{\mathbf{B}} \cdot \tilde{\mathbf{k}}) \tilde{u}_{z}+i \tilde{u}_{x}\left(\tilde{B}_{z}-q \frac{\partial \tilde{B}_{z}}{\partial \tilde{x}}\right)$.

Using the Eqs. (13), together with the Eqs. (14), we obtain a differential equation for the total pressure perturbation

$-(1+\tilde{x}) \frac{\partial^{2} \tilde{p}}{\partial \tilde{x}^{2}}+D \frac{\partial \tilde{p}}{\partial \tilde{x}}+F \tilde{p}=0$,

where

$q=1+\tilde{x}, \quad S_{1}=\frac{(\tilde{\mathbf{B}} \cdot \tilde{\mathbf{k}})^{2}}{q \tilde{\omega}}-q \tilde{\rho^{*}} \tilde{\omega}$, 


$$
\begin{aligned}
& L=-2 \frac{(\tilde{\mathbf{B}} \cdot \tilde{\mathbf{k}})^{2}}{q \tilde{\omega} S_{1}}, \quad G=\tilde{B}_{y} \frac{\partial \tilde{B}_{y}}{\partial \tilde{x}}+\tilde{B}_{z} \frac{\partial \tilde{B}_{z}}{\partial \tilde{x}}, \\
& S=S_{1}+\frac{2}{q \tilde{\omega}}\left(\tilde{B}^{2}-q G\right)+L \frac{2 \tilde{B}^{2}}{q \tilde{\omega}}, \\
& Q=-\frac{1}{q}(2+L), \quad D=-1+L+(1+\tilde{x})\left[\frac{1}{S} \frac{\partial S}{\partial \tilde{x}}+Q\right], \\
& F=\tilde{k}^{2} \frac{S}{q S_{1}}+\frac{\partial L}{\partial \tilde{x}}-\frac{L}{S} \frac{\partial S}{\partial \tilde{x}}-Q L .
\end{aligned}
$$

For the Eq. (15), we have the following boundary conditions:

$\tilde{x}=\tilde{x}_{1}: \frac{\partial \tilde{p}}{\partial \tilde{x}}=-\kappa_{1} \tilde{p} ; \quad \tilde{x}=\tilde{x}_{2}: \frac{\partial \tilde{p}}{\partial \tilde{x}}=\kappa_{2} \tilde{p}$.

Thus, a second order ordinary differential Eq. (15) has to be solved for the perturbation of the total pressure with boundary conditions (Eq. 16) on either side of the layer. The coefficients of this equation are functions of the frequency and the wave number. Using the different functions for the magnetic field variation across the magnetopause, this equation is integrated numerically, and the frequency is determined by satisfying the boundary condition.

In order to clarify the driving term of the instability, we analyze Eq. (15) in the case of large $k a$. In this case we can neglect the term with the first derivative of $\tilde{p}$ in Equation (15), which is simplified to

$-\frac{\partial^{2} \tilde{p}}{\partial \tilde{x}^{2}}+k^{2} \frac{1}{q}\left[1-\frac{1}{q^{2} \tilde{\rho}^{*} \omega^{2}}\left(2 \tilde{B}^{2}-q \frac{\partial \tilde{B}^{2}}{\partial \tilde{x}}\right)\right] \tilde{p}=0$.

Using zero conditions for $\tilde{p}$ and assuming $\tilde{\omega}=i \tilde{\gamma}$ (where $\gamma$ is the growth rate), we multiply Eq. (17) with the complex conjugate function $\hat{\tilde{p}}$ and integrate it in the range from $(-\infty ;+\infty)$. After these operations, finally, we obtain the equation

$$
\begin{aligned}
& \int_{-\infty}^{+\infty}|\partial \tilde{p} / \partial \tilde{x}|^{2} d \tilde{x}+k^{2} \int_{-\infty}^{+\infty}|\tilde{p}|^{2} \frac{1}{q} d \tilde{x}+ \\
& k^{2} \int_{-\infty}^{+\infty} \frac{1}{q^{3} \tilde{\rho}^{*} \gamma^{2}}\left(2 \tilde{B}^{2}-q \frac{\partial \tilde{B}^{2}}{\partial \tilde{x}}\right)|\tilde{p}|^{2} d \tilde{x}=0 .
\end{aligned}
$$

Here, the growth rate $\gamma$ is assumed to be real. If $\partial B^{2} / \partial x \leq 0$, then all terms in Eq. (18) will be positive. In such a case this equation yields the trivial result $\tilde{p}=0$, and thus, the instability is not possible.

Therefore, for the interchange instability to appear, it is necessary to have a region where the $x$ derivative of the magnetic pressure is positive, $\partial B^{2} / \partial x \geq 0$. Hence, the driving term for the instability is a positive magnetic pressure gradient, and, correspondingly, a negative plasma pressure gradient in the $x$ direction. This means that the instability takes place in the subsolar magnetosheath if there exists a local region with the plasma pressure decreasing along the $x$ axis which is directed to the Sun.
A qualitative physical explanation of the interchange instability is the following: Considering a positive gradient of the background magnetic pressure $\left(\partial B^{2} / \partial x \geq 0\right)$, let us push slightly a thin magnetic flux tube which was initially in equilibrium. For this tube being pushed forward along the $x$ axis, the equilibrium will be broken, and the surrounding plasma pressure will become less than that inside the tube. A disbalance will appear between the positive force provided by the gradient of the total pressure and the negative force caused by the magnetic tension that is directed towards the curvature center. The resulting force will be positive and thus, the magnetic tube will be further accelerated along the $x$ direction.

\section{Results}

To clarify the influence of different parameters of the layer on the interchange instability, we first analyze the case of an analytical monotonic variation of the magnetic field between two values $\tilde{B}_{m}\left(\tilde{x}=\tilde{x}_{2}\right)$ and $\tilde{B}_{s}\left(\tilde{x}=\tilde{x}_{1}\right)$. We suppose that the magnetic fields $\tilde{B}_{m}$ and $\tilde{B}_{s}$ are antiparallel. The model profile of the magnetic field in dimensionless form is,

$$
\tilde{B}=-\frac{\left(\tilde{B}_{s}+\tilde{B}_{m}\right)}{2} \tanh \left[\frac{5}{\tilde{a}}\left(\tilde{x}-\tilde{x}_{0}\right)\right]+\frac{\tilde{B}_{m}-\tilde{B}_{s}}{2},
$$

where $\tilde{x}_{0}=\left(\tilde{x}_{1}+\tilde{x}_{2}\right) / 2$. This model is very convenient for analyzing the dependence of the instability growth rate on the different parameters; as the curvature radius, the wave number, and the layer thickness. The instability growth rate, magnetic field, and plasma parameters are normalized in accordance to Eq. (12). The dimensionless magnetic field, which is scaled to $\sqrt{4 \pi \rho_{s w}} U_{s w}$, is assumed to vary from $\tilde{B}_{m}=1.3\left(\tilde{x}_{2}=1\right)$ to $\tilde{B}_{s}=1.17\left(\tilde{x}_{1}=\tilde{x}_{2}+\tilde{a}\right)$, where $\tilde{a}=a / R, a$ is the thickness of the layer.

These values are chosen to be suitable for the subsolar magnetopause. The first value 1.3 is related to the geomagnetic field at the subsolar point which can be estimated from the pressure balance condition,

$$
B_{m}^{2} /(8 \pi)=C \rho_{s w} U_{s w}^{2} .
$$

The second value is related to the magnetic barrier close to the magnetopause, where the magnetic pressure is enhanced, but it is slightly less than the total pressure estimated as $C \rho_{s w} U_{s w}^{2}$. Here, $U_{s w}$ is a speed of the solar wind, and the coefficient $C$ is equal approximately 0.9 (see Spreiter et al., 1966).

Figure 3 illustrates the geometrical situation for the first case. Shown is the interchange instability of a fixed curved layer between antiparallel magnetic fields with a smooth variation of the magnetic vector. From the numerical solution, the instability growth rate is found as a function of the layer thickness, curvature radius, and wave number.

Figure 4 shows the growth rate of the interchange instability (in units $\gamma^{*}=U_{s w} / R$ ) as a function of the dimensionless 


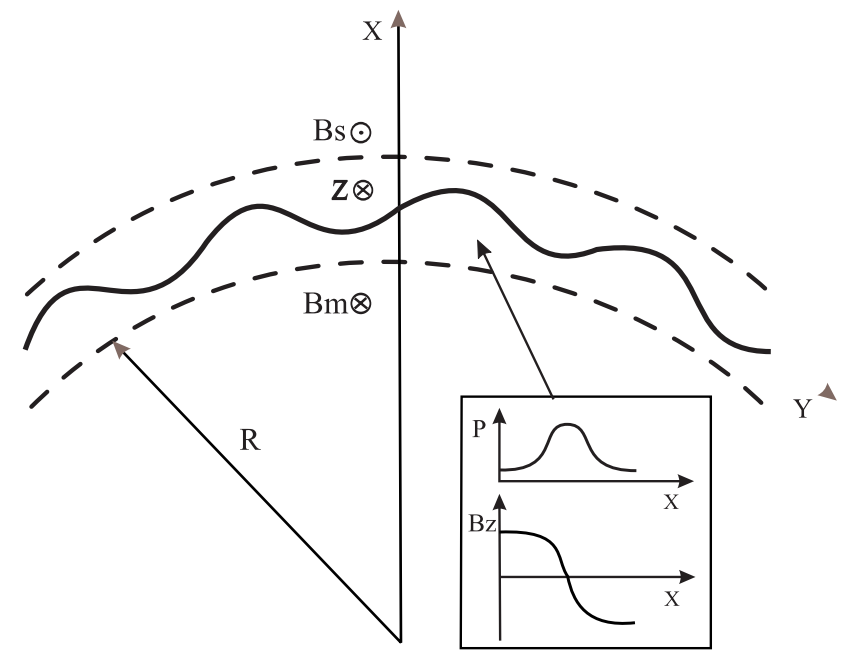

Fig. 3. Illustration for the first case with a fixed curved layer with reversal magnetic fields.

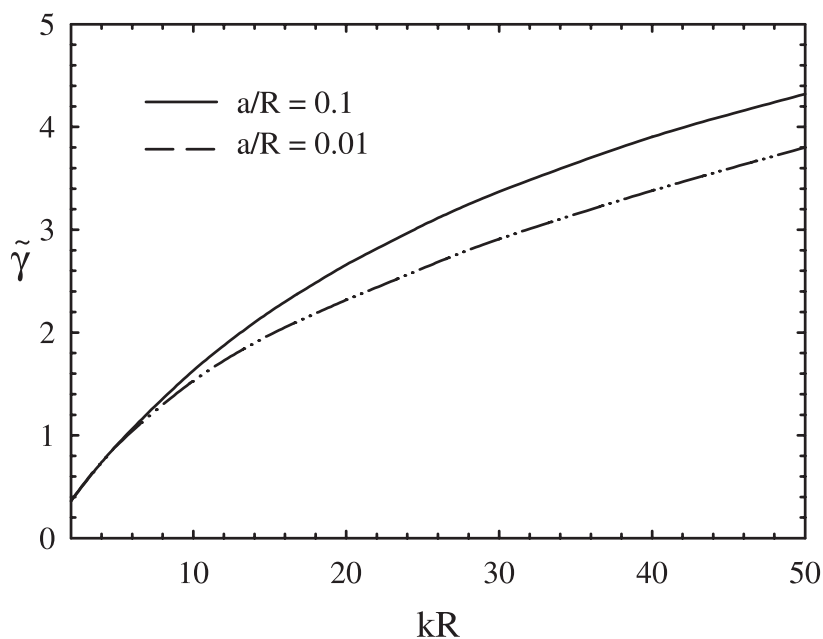

Fig. 4. Normalized instability growth rate as a function of $k R$ for the model profile of the magnetic field. Different curves correspond to different values of $a / R=0.1 ; 0.01$.

parameter $k R$ for two different ratios of the layer thickness and the curvature radius, $a / R=0.1,0.01$. This figure indicates that the instability growth rate is larger when the perturbation wavelength is shorter, and the layer is thicker. From the normalization it follows that the physical value of the growth rate $\gamma$, as well as the magnetic field strength at the layer boundary $B_{m}$, are proportional to $U_{s w}$, and thus, $\gamma \propto B_{m}$.

The second case considered in our work is that for the instability of a thin layer convected from the bow shock to the magnetopause. This layer is related to a nonsteady IMF variation in the solar wind. For this layer, the profiles of magnetic field, plasma pressure and density are varying slowly in time because of the layer motion to the magnetosheath. And correspondingly, the instability growth rate is depending on the position of the layer.

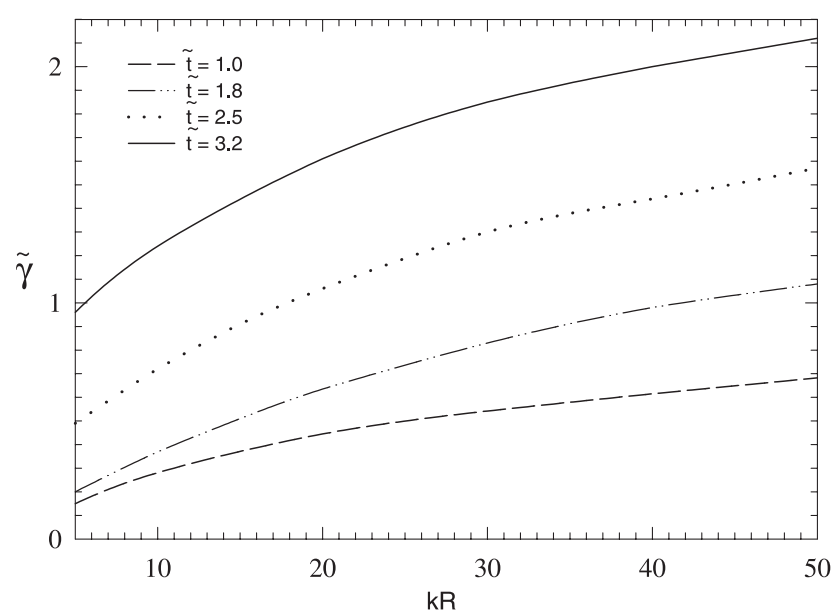

Fig. 5. Normalized instability growth rate as a function of $k R$ for the nonsteady magnetosheath profiles corresponding to different times: $t=1.0 ; 1.8 ; 2.5 ; 3.2$.

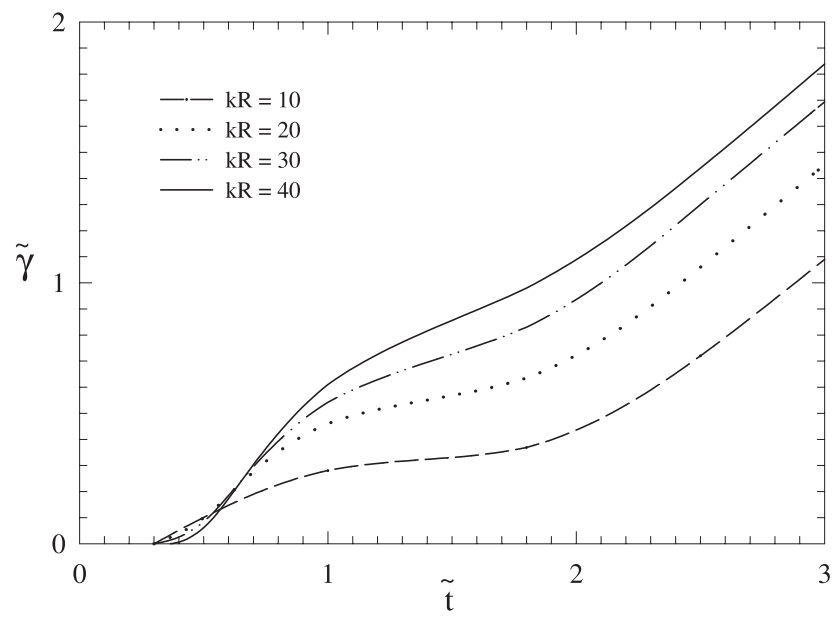

Fig. 6. Normalized instability growth rate as a function of time for the nonsteady magnetosheath profiles corresponding to different wave numbers $k R=10 ; 20 ; 30 ; 40$.

Figure 1 shows the geometrical situation for the second case. A curved layer with antiparallel magnetic fields is moving from the bow shock towards the magnetopause. For our instability analysis, we use the profiles of the plasma density, magnetic field, and plasma pressure which are shown in Fig. 2.

Figure 5 shows the normalized growth rate of the interchange instability as a function of the wave number $k R$ for different positions of the current layer during its motion in the magnetosheath from the bow shock towards the magnetopause. Different curves are obtained for the numerical profiles corresponding to different normalized times $t=1.0 ; 1.8$; 2.5; 3.2 (in units $R / U_{s w}$ ). It is evident that the instability growth rate is a monotonic increasing function of the wave number. For larger time, this monotonic dependence becomes stronger. Taking a reasonable value $R=10 R_{E}$ for the 
Earth's magnetosheath, we estimate the following range of wave numbers related to the figure, $0.5 / R_{E} \leq k \leq 5 / R_{E}$. This range corresponds to the scales of perturbations estimated as $1 . R_{E} \leq \lambda \leq 2 R_{E}$. For the same layer, the instability growth rate is also shown in Fig. 6 as a function of the convection time (in units $R / U_{s w}$ ). The different curves are corresponding to the different wave numbers $k R=10 ; 20 ; 30 ; 40$. One can see that the largest instability growth rate corresponds to the shortest scales of perturbations. The figure indicates that the maximal growth rate of the instability is about $2 U_{s w} / R$, where $R$ is a curvature radius of the subsolar magnetopause.

The assumption of an incompressible plasma as used in our model, can be justified as follows: Perturbations of the density propagate with the fast magnetosonic speed $c_{s+}$. The incompressible MHD model can be applied when the distance of the compressible wave propagation exceeds the wave scale of the interchange mode, $c_{S} \tau \gg 1 / k$ (where $c_{S+}$ is the fast magnetosonic speed and $\tau=\gamma^{-1}$ ). The fast magnetosonic speed is higher than the Alfvén speed, and thus, the last condition is covered by the more strong condition $V_{a} \tau \gg 1 / k$, which can be written in dimensionless form,

$\left(V_{a} / U_{s w}\right)(k R) \tilde{\tau} \gg 1$.

Inside the magnetic barrier, there is $B \approx \sqrt{8 \pi \rho_{s w}} U_{s w}$, $\rho \approx \rho_{s w}$, and thus, $V_{a} / U_{s w} \approx 1$. Finally, the condition (20) is simplified to $(k R) \tilde{\tau} \gg 1$, which is fulfilled because $\tilde{\tau} \geq 0.5$ and $k R \gg 1$.

\section{Discussions and conclusions}

The growth rate of the interchange instability is studied for a thin curved layer with smooth variations of plasma parameters and reversal magnetic field structure. There are five main factors providing an enhancement of the interchange instability growth rate for the layer: 1) Increase in the thickness of the layer; 2) Increase in the magnetic field strength on the both sides of the layer; 3) Decrease in the plasma density; 4) Increase in the wave number; 5) Decrease in the local curvature radius of the layer.

In particular, the interchange instability is analyzed for the layer in the Earth's magnetosheath which is caused by a nonsteady variation of the interplanetary magnetic field from north to south. For a steady-state solar wind flow around the magnetosphere, the magnetic field is enhanced near the magnetopause in the boundary layer, which is called magnetic barrier or plasma depletion layer (see Erkaev et al., 2000 and references therein). In the case of a nonsteady IMF variation, we analyze the behaviour of a thin layer which is moving on the background of the magnetic barrier. The interchange instability growth rate is obtained which is increasing monotonically while the layer is approaching towards the magnetopause. This monotonic behavior of the growth rate is related to an enhancement of the magnetic field strength in the magnetic barrier in the course of the layer moving towards the magnetopause. This instability has a pure imaginary fre- quency and thus, the wave perturbations do not propagate, instead they are just transported with a bulk plasma flow.

Using the maximal growth rate from Figs. 6, 7 and taking the solar wind velocity $U_{s w}=500 \mathrm{~km} / \mathrm{s}$, and a radius of the magnetosphere $R=10 R_{E}$, we estimate the instability growth time as $\tau=1 / \gamma \sim 0.5 R / U_{s w} \sim 60 \mathrm{~s}$. This time is short enough, and thus, the instability can evolve into a nonlinear stage.

In our model we consider a reversal time scale of about $1 \mathrm{~min}$. This is consistent with observations of Horbury et al. (2001). The reversal length scale is dependent on the position of the convecting current sheet with respect to the magnetopause. In our case, it decreases along the subsolar line from the value of about $1 R_{E}$ near the bow shock to a value of $0.1 R_{E}$ near the magnetopause.

We consider a fully reversed field current layer, for simplicity. In such a case we have the largest growth rate. For a nonlocal analysis with smooth profiles, it is rather complicated to take into account a shear angle. However, effects of a finite shear angle have been studied previously by Arshukova et al. (2002) for the particular case of a uniform layer with enhanced plasma pressure which is separated by two tangential discontinuities. The interchange instability growth rate was obtained by Arshukova et al. (2002) as a function of the magnetic shear angle. This growth rate was found to be positive for a finite interval of the shear angle.

It is important to note that the interchange instability might also take place for convected tangential discontinuities in the magnetosheath with monotonic variations of the magnetic field decreasing towards Earth.

Smooth magnetic field variations occurring under a constant total pressure might be the reasons for the corresponding variations of plasma pressure and density. Wide and smooth variations of the density can develop into sharp and relatively smallscale perturbations in the magnetic barrier.

A nonlinear behaviour of the unstable interchange modes in the magnetosheath can be described qualitatively as follows: The instability will destroy the structure of a tangential discontinuity, and finally, it will result in the appearance of two kinds of thin magnetic flux tubes: a) magnetic tubes with enhanced plasma pressure with respect to the ambient medium; b) magnetic tubes with decreased plasma pressure. The scale of these tubes is expected to be of about $500 \mathrm{~km}$. The first type of tube could have a zero velocity because of the equilibrium of two forces: The first force directed opposite to the magnetopause is provided by a disbalance between the total pressure gradient and the magnetic tension; The second force is a hydrodynamic one provided by the ambient plasma flow towards the magnetopause. The second kind of tube is just convected downstream with the ambient plasma. This is a possible qualitative explanation of the existence of slow modes of the enhanced plasma pressure and density in the subsolar magnetosheath, which look similar to the socalled "slow mode transition regions" observed in the magnetosheath (Song et al., 1992). 
Acknowledgements. This work is supported by the INTAS-ESA project 99-01277, by grants No 01-05-65070, No 02-05-06151, and No 03-05-06101 from the Russian Foundation of Basic Research, by grant E02-8.0-22 from the Russian Ministry of Higher Education, by project I.4/2001 from "Österreichischer Austauschdienst". It is also supported in part by the Austrian "Fonds zur Förderung der wissenschaftlichen Forschung" under project P13804-TPH. We acknowledge support by the Austrian Academy of Sciences, "Verwaltungstelle für Auslandsbeziehungen", and the Russian Academy of Sciences.

Topical Editor T. Pulkkinen thanks two referees for their help in evaluating this paper.

\section{References}

Alexandrov, A. F., Bogdankevich, L. S., and Rukhadze, A. A.: Principles of Plasma Electrodynamics, Springer-Verlag, 1984.

Alexeev, I. I. and Maltsev, Y. P.: Estimation of coefficient of IMF penetration in the magnetosphere as a result of developement of interchange instability, Geomagnetism and Aeronomy, 30, 134, 1990.

Arshukova, I. L. and Erkaev, N. V.: Interchange instability of the subsolar magnetopause, in: Solar Wind - Magnetosphere 3, edited by Biernat, H. K., Farrugia, C., and Vogl, D., Österreichische Akademie der Wissenschaften, Vienna, 43, 2000.

Arshukova, I. L., Erkaev, N. V., and Biernat, H. K.: Magnetohydrodynamic instability of a high magnetic shear layer with a finite curvature radius, Phys. Plasmas, 9, No 2, 401, 2002.

Chandrasekhar, S.: Hydrodynamic and hydromagnetic stability, Oxford University Press, London, 1968.
Erkaev, N. V., Farrugia, C. J., Biernat, H. K., Burlaga, L.F., and Bachmaier G.A.: Ideal MHD flow behind interplanetary shocks driven by magnetic clouds, J. Geophys. Res., 100, 19919, 1995.

Erkaev, N. V., Biernat, H. K., and Farrugia, C. J.: Ideal magnetohydrodynamic flow around a blunt body under anisotropic pressure, J. Geophys. Res., 7, 3413, 2000.

Erkaev, N. V., Farrugia, C. J., and Biernat, H. K.: The role of magnetic barrier in the solar wind- magnetosphere interaction, Planet. Space Sci., 51, 745, 2003.

Farrugia, C. J., Erkaev, N. V., Biernat, H. K. and Burlaga, L. F.: Anomalous magnetosheath properties during passage of an interplanetary magnetic cloud, J. Geophys. Res., 100, 19245, 1995.

Freidberg, J. P.: Ideal Magnetohydrodynamics, Plenum Press, New York, 1987.

Horbury, T., Burgess, D., Fränz, M., and Owen, C. J.: Three Spacecraft Observations of Solar Wind Discontinuities, Geophys. Res. Lett., 28, 677, 2001.

Landau, L. D. and Lifshitz, E. M.: Electrodynamics of Continuous Media, Pergamon Press, Oxford, 1960.

Petrinec, S. M. and Russell, C. T.: Hydrodynamics and MHD equations across the bow shock and along the surfaces of planetary obstacles, Space Sci. Rev., 79, 757, 1987.

Rezenov, B. V. and Maltsev, Y. P.: Role of interchange instability in flux transfer event origin, Ann. Geophys., 12, 183, 1994.

Song, P. C., Russel, C. T., and Thomsen, M. F.: Slow mode transition in the frontside magnetosheath, J. Geophys. Res., 97, 8295, 1992.

Spreiter, J., Summers, A. L., and Alskne, A. Y.: Hydrodynamic flow around the magnetosphere, Planet. Space Sci., 14, 223, 1966. 